\title{
The RB tumor suppressor: a gatekeeper to hormone independence in prostate cancer?
}

\author{
Kay F. Macleod \\ Ben May Department for Cancer Research, Gordon Center for Integrative Sciences, University of Chicago, Chicago, Illinois, USA.
}

\begin{abstract}
The retinoblastoma tumor suppressor gene ( $R B 1$; encoding $R B)$ is often cited as a gatekeeper, whose inactivation - direct or indirect - is a rate-limiting step for tumor initiation. However, in this issue of the JCI, Sharma et al. show that $R B 1$ loss is a late event in human prostate cancer that is coincident with the emergence of castrate-resistant metastatic disease. This role for $R B 1$ was linked to both E2F transcription factor 1-driven upregulation of the androgen receptor (AR) and increased recruitment of the $A R$ to target gene promoters. This unexpected function for $R B 1$ in late-stage cancer calls upon us to reassess the significance of $R B 1$ inactivation in other cancers in terms of its timing, function in disease etiology, and relevance for cancer therapy.
\end{abstract}

\section{The retinoblastoma pathway paradigm is challenged}

The canonical function of the retinoblastoma protein (RB; encoded by RB1), elucidated primarily through the manipulation of human tumor cell lines and genetically engineered mouse models, has established a key role for $\mathrm{RB}$ in repressing the transcriptional activity of the so-called activator class of E2F transcription factors (E2F1-E2F3) (1, 2). E2Fs regulate the expression of a growing number of genes but are best defined for their transcriptional regulation of genes required for cell cycle, including genes involved in DNA replication, nucleotide synthesis, and checkpoint control. Initial work indicated that regulation of E2F activity by $\mathrm{RB}$ occurred primarily at the transition between the G1 and $S$ phases of the cell cycle. However, there is growing appreciation that $\mathrm{RB}$ can be activated in S-phase by dephosphorylation in response to stresses, such as oxidative stress, and that $\mathrm{RB}$ regulates the mitotic checkpoint by modulating the expression of E2F target genes involved in chromosome segregation and cytokinesis (2).

The phosphorylation state and activity of $\mathrm{RB}$ is sensitive to environmental cues mediated largely through the activity of cyclindependent kinase 4 (CDK4) and CDK6 complexed to D-type cyclins. Phosphorylation of $\mathrm{RB}$ on key residues inhibits binding to E2Fs and also derepresses CDK2 complexed to E-type cyclins, resulting in further RB phos-

Conflict of interest: The author has declared that no conflict of interest exists.

Citation for this article: J Clin Invest. 2010; 120(12):4179-4182. doi:10.1172/JCI45406. phorylation, E2F target gene activation, S-phase entry, and DNA replication origin firing. The p16/INK4A tumor suppressor acts upstream to promote RB dephosphorylation and activation by directly inhibiting CDK4 and CDK6 and indirectly inhibiting CDK2 through release of $\mathrm{p} 21 / \mathrm{CIP} 1$ and $\mathrm{p} 27 /$ KIP1. Based on nonoverlapping patterns of inactivation of RB and p16/INK4A in human cancers, it was proposed that loss of p16/INK4A obviated the need to inactivate RB (3) and vice versa. It was further suggested that general inactivation of the RB pathway (Figure 1) and deregulation of the cell cycle was a common early event in human cancers that could be achieved in various different ways (exemplified by translocation of cyclin D1 in mantle cell lymphoma, deletion of p16/INK4A in melanoma, deletion of RB1 in osteosarcoma, upregulation of Bmi-1 in medulloblastoma, and amplification of $E 2 F 3$ in bladder cancer) (1). In this issue of the JCI, Sharma and colleagues challenge this simple paradigm with data that suggest that unique functions of RB in tumorigenesis, particularly in advanced stages of cancer, need to be further examined (4).

\section{Insight into the etiology of castrate- resistant prostate cancer}

In 1966, Charles B. Huggins was awarded the Nobel Prize for Medicine for his landmark discovery that hormone deprivation as a result of castration induced rapid regression of prostate cancer (5), with subsequent studies showing that this was likely due to apoptosis of androgen-dependent tumor cells and tumor involution (6). However, tumors generally recur as aggressive castrate-resistant prostate cancer (CRPC) that grows independently of androgen in a manner that has been variously attributed to upregulation of AR levels caused by $A R$ gene amplification, expression of androgens by tumor cells, and gain-of-function mutations that alter AR stability or affinity for ligand (6). The work of Sharma and colleagues (4) suggests that AR upregulation in CRPC is causally linked to loss of the RB tumor suppressor, resulting in derepression of E2F1 and altered activity of AR at specific target genes. Using an extensive set of primary human prostate cancer samples, the authors demonstrated that whereas RB1 mRNA expression was intact in prostate carcinoma in situ, it was lost in a significant proportion of metastatic CRPCs. These observations are supported by the selective induction of the RB loss gene expression signature in advanced prostate tumors. RB levels also showed reduced expression in CRPC, which the authors suggest may be attributed to allelic loss at the RB1 locus. These data are consistent with recent comparative genome hybridization (CGH) analyses of human prostate cancer showing that $R B 1$ loss is preferentially observed in advanced prostate cancer (7). While stable knockdown of RB levels in human prostate tumor cell lines did not markedly affect tumor growth in xenografted male mice prior to castration, Sharma et al. observed a dramatic effect of RB loss on tumor growth following castration (4). This effect was manifested by diminished reduction in tumor burden following castration and by faster recovery in tumor mass and serum prostate-specific antigen (PSA) levels.

Sharma and colleagues (4) attribute these castration-dependent effects of RB loss to the marked increase in the expression of $A R$ protein and mRNA observed when RB was knocked down in both cultured cell lines and xenografted tumors. That these effects were more striking following castration of host mice suggests that loss of androgen signaling activates RB, although whether this is achieved through dephosphorylation or other mechanisms was not investigated. 


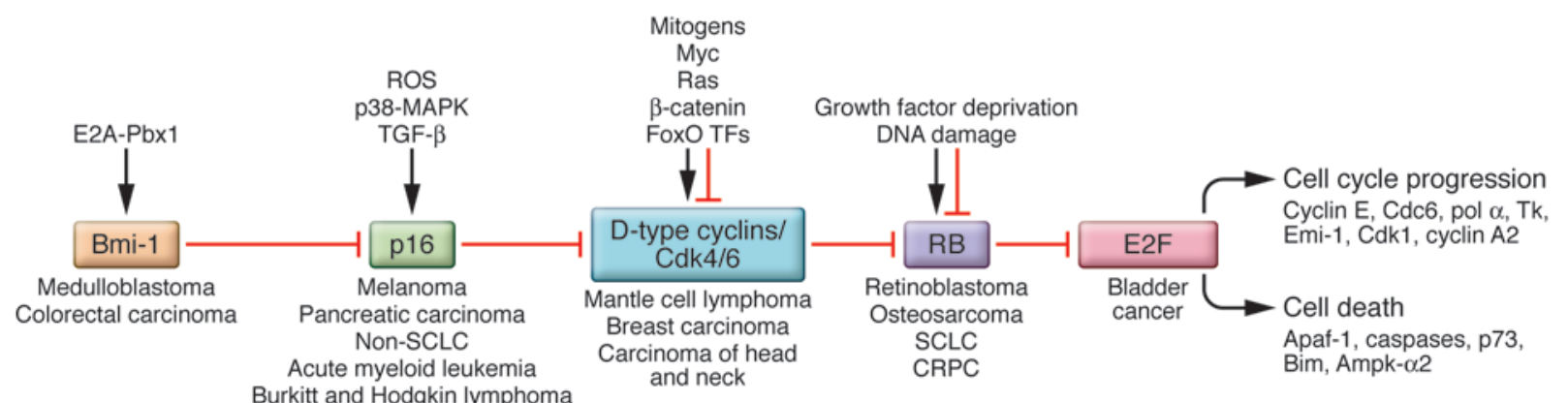

\section{Figure 1}

Inactivation of the RB pathway in human cancers. Different components of the RB pathway are deregulated in human cancers, with the overall effect of derepressing E2F transcription factors that promote cell cycle progression or programmed cell death. When cell death pathways are inactivated, as is the case in most cancers, activation of E2Fs drives proliferation through induction of target genes such as cyclin E, CDC6, and CDK1. Loss of the RB1 tumor suppressor is prevalent in osteosarcoma and small-cell lung carcinoma (SCLC) in addition to its defining loss in human retinoblastoma, but it was initially confounding that its loss was not more commonly detected. However, with the discovery that p16/INK4A functions as an upstream inhibitor of CDK4 and CDK6 and thus as an activator of RB, and that loss of the p16/INK4A locus occurred in many of the cancers where RB1 loss was not detected, Weinberg and others proposed that functional inactivation of RB could be achieved in different tumors through targeting different components of the RB pathway (16). Thus, loss of $p 16 / I N K 4 A$ in melanoma, translocation of cyclin D1 in mantle cell lymphoma, or, indeed, overexpression of BMI-1 in medulloblastoma has been proposed to have similar functional readout to RB loss in terms of E2F activity and cellular proliferation. Ampk- $\alpha 2$, $\alpha 2$ subunit of AMPK; Apaf-1, apoptosis protease activating factor-1; E2A$\mathrm{Pbx} 1$, the translocation product of fusion of the E2A gene to the Pbx1 gene; Emi-1, early mitotic inhibitor 1; FoxO TFs, forkhead box O subclass of transcription factors; Tk, thymidine kinase; pol $\alpha$, DNA polymerase- $\alpha$.

Elevated expression of the AR is a key feature of CRPC, and Sharma and colleagues provide compelling evidence that $A R$ is an E2F1 target gene that is cell cycle regulated (4). Importantly, AR levels were specifically elevated in primary human prostate cancers that had lost $R B 1$. Loss of RB also increased the recruitment of the AR to cognate promoters, resulting in increased AR target gene expression. Thus, the work of Sharma and colleagues identifies, for the first time to my knowledge, two distinct effects of RB loss in CRPC: (a) increased AR expression mediated by E2F1, and (b) increased AR target gene expression mediated by the AR. RB-mediated repression of both the expression and the activity of the AR may thus explain selection for $R B 1$ loss during progression to CRPC.

\section{Does loss of RB potentiate E2F1- regulated expression and activity of the AR?}

Sharma and colleagues show that key AR target genes, such as PSA and transmembrane protease, serine 2 (TMPRSS2), are elevated in RB-deficient cells and tumors and, importantly, that loss of RB increases AR binding to the promoter and enhancer of the PSA gene, both in the presence and absence of androgen (4). Intriguingly, RB loss also increased recruitment of AR to key cell cycle genes recently identified as bona fide AR targets, including CDK1 and cyclin A2 (8). Many of these genes are also
E2F targets, and these observations raise the interesting question of how E2Fs and the AR might coregulate cell cycle genes. Significantly, AR-mediated transcriptional upregulation of these mitotic genes is specific to CRPC, with AR conversely implicated in suppression of proliferation in normal prostate epithelium (8). Does loss of RB in CRPC promote synergistic interaction of E2F1 with the AR at the promoters of these mitotic cell cycle genes, and does this explain the differential effect of the AR on cell cycle gene expression in CRPC versus normal prostate epithelium? Are there other non-cell cycle target genes that are coregulated by E2F1 and the AR that play a role in invasion and metastasis or other processes relevant to CRPC? These are all key questions raised by the important new work of Sharma and colleagues (4).

The observed clinical correlation between levels of AR mRNA and E2F1 mRNA, but not E2F3 mRNA, in primary CRPC is consistent with the central role attributed by Sharma et al. to E2F1 in regulating the AR (4). However, this was perhaps an unexpected conclusion, particularly since E2F3 has previously been implicated in prostate cancer, where it has been shown to be overexpressed (9). The unique role for E2F1 in the regulation of AR and progression to CPRC may reflect its relative affinity for phosphorylated RB in CRPC compared with other E2Fs, or it may indeed suggest a specific interaction of E2F1 with
AR at particular target genes, or E2F1 may participate in unique interactions with coactivators required for AR-dependent transactivation, or a combination of all of the above. This key role played by E2F1 in regulating $A R$ also makes it unlikely that the RB-related proteins p107 and p130 compensate for RB loss in late-stage prostate cancer, since these RB-related pocket proteins preferentially interact with E2F4 and E2F5 and have weaker affinity for E2F1 (10). Consistent with this, neither p107 nor p130 expression levels appeared to be altered in primary human prostate cancer (4), and the authors showed that in contrast to knockdown of RB, knockdown of either p107 or p130 failed to alter AR expression in prostate cancer cell lines, although, arguably, knockdown of both p107 and p130 together might be required to resolve this more convincingly.

\section{Piecing it all together - reflections on the clonal origins of CRPC}

A major question arising from the work of Sharma and colleagues (4) is how the cell cycle is deregulated to promote proliferation at early intraepithelial stages in prostate cancer if RB is functionally intact. Does Myc overexpression, as detected in early stages of prostate cancer (6), promote increased CDK activity to drive proliferation in prostatic intraepithelial neoplasia (PIN)? Is CDK activity later turned off in response to DNA damage signals emanating from telomere 


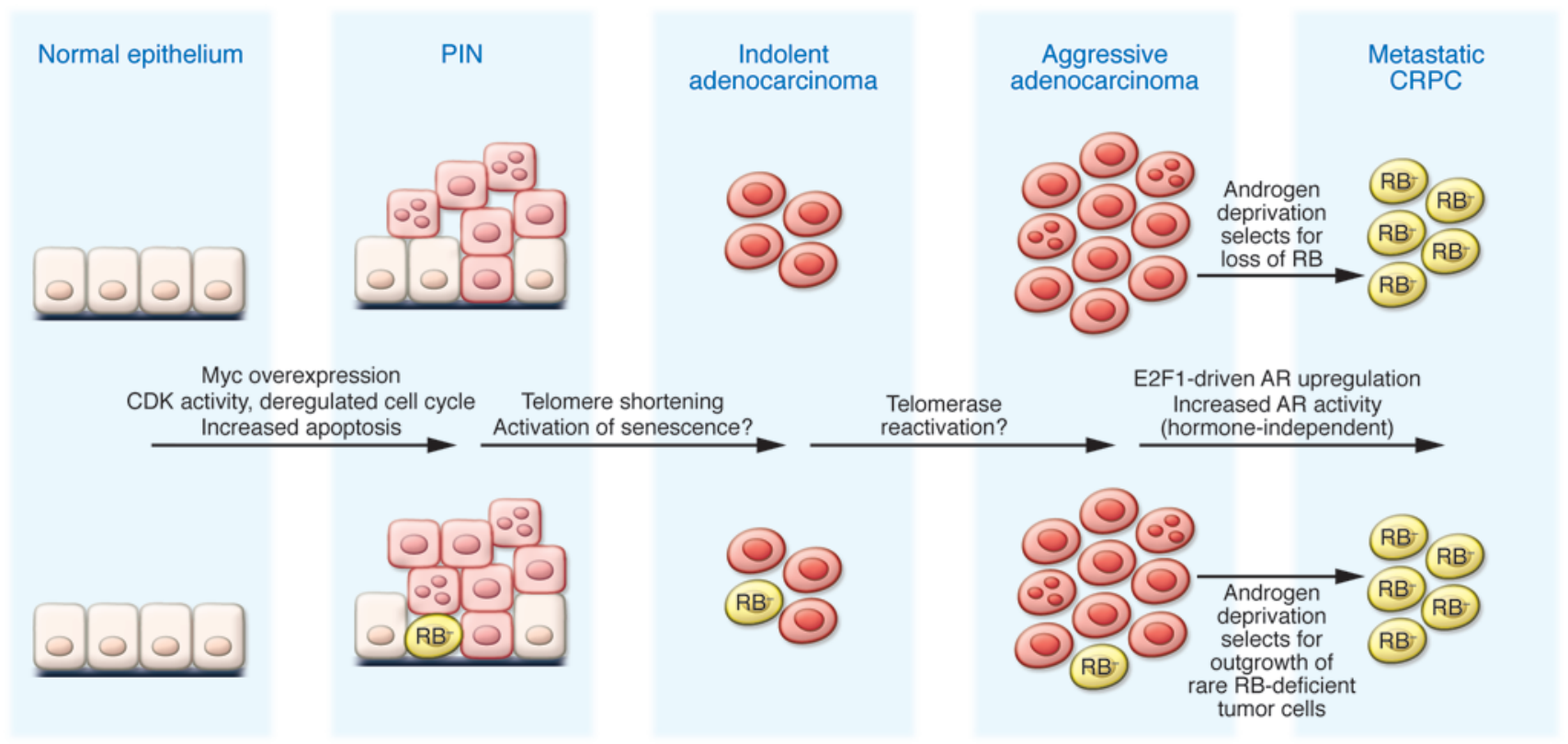

Figure 2

Understanding the role of RB in preventing progression to CRPC at the cellular level. The progression of prostate cancer through different stages, from PIN to adenocarcinoma to metastatic CRPC, has been associated with activation of specific oncogenes, such as Myc, and loss of key tumor suppressors, such as PTEN, at early stages of tumorigenesis (6). Detectable loss of RB1 at late stages upon progression to CRPC raises the intriguing question as to whether androgen deprivation is selecting for androgen independence through genetic loss/epigenetic silencing of RB1 in previously androgen-dependent cells, or whether such RB-deficient androgen-independent cells were present in low abundance throughout tumorigenesis, but now have a growth advantage over RB-proficient androgen-dependent cells, allowing them to expand to become the dominant tumor cell type represented.

shortening, or does this occur by androgen blockade or castration to allow RBmediated repression of the AR (Figure 2)? Or is hyperphosphorylated RB capable of repression of the AR through E2F1, as was recently shown for repression of proapoptotic genes by RB (11), even if it cannot repress cell cycle genes? Alternatively, do these observations support the clonal selection model for prostate cancer progression to CRPC, in which androgen deprivation selects for rare RB-deficient androgenindependent cells that can proliferate and survive in the absence of androgens (Figure 2)? This is a challenging question to answer, but the work by Sharma et al. (4) may provide a new handle on determining the molecular origins of CRPC.

While Sharma and colleagues identify upregulation of the AR and hormone-independent growth as a major consequence of RB loss at late stages of tumorigenesis (4), one also has to consider the selective pressures that may be acting to promote retention of the $R B 1$ gene during the early stages of prostate cancer, especially given its apparent pattern of early inactivation in other cancers. Does the antiapoptotic function of RB select against its inactivation at early stages of prostate cancer tumorigenesis? If so, this would suggest that the cell cycle and apoptosis functions of RB are uncoupled in early-stage prostate cancer. Or is $\mathrm{RB}$ required for the cellular senescence that is implicated as a tumor suppressor mechanism at the adenocarcinoma stage of prostate cancer progression? The findings of Sharma et al. indicate that RB acts in a more nuanced manner than currently appreciated and indeed point to the importance of considering the stage of tumor progression when addressing RB function (4).

\section{Significance for treatment of CRPC}

New therapeutic agents have recently been developed that bind the AR with greater affinity than more commonly used antiandrogen drugs, such as flutamide or bicalutamide (12). These new second-generation antiandrogens prevent the AR from binding to its cognate recognition site in target gene promoters, in part by interfering with its nuclear translocation, but also by preventing its interaction with transcriptional coactivators. Conversely, Sharma et al. showed that RB loss promoted recruitment of the AR to its target genes in the presence or absence of androgen blockade with bicalutamide (4), and it will therefore be interesting to determine how RB loss affects the therapeutic efficacy of RD162 and MDV3100, two of the new antiandrogens. Also, should RB status be used as a metric to direct hormone therapy to responsive patients? Or, conversely, does chemical or surgical castration select for upregulation of the AR as a result of $\mathrm{RB}$ loss, as discussed above? Along these lines, further work is required to determine what mechanisms give rise to $\mathrm{RB}$ inactivation in CRPC. Does hemizygous deletion of RB followed by gene conversion of the second allele take place in CRPC, in alignment with Knudson's two-hit hypothesis (13) for early-stage loss of RB in human retinoblastoma? Or are alternative mechanisms, such as epigenetic silencing, taking place? Provocative data in the report by Sharma et al. (4) showing inconsistent expression of $\mathrm{RB}$ protein and mRNA in tumors with at least one allele of $R B 1$ intact suggest that such alternative inactivation mechanisms may be at play. These questions remain unanswered, but may be relevant to identifying therapeutic interventions aimed at reactivating $\mathrm{RB}$ to prevent progression of prostate cancer to castration resistance or 
indeed to promote sensitivity to antiandrogen therapy at late stages.

\section{Nuclear hormone receptor regulation by RB/E2F in other cancers}

Finally, if RB modulates the expression and activity of AR in prostate cancer, are other nuclear hormone receptors regulated by $\mathrm{RB} / \mathrm{E} 2 \mathrm{Fs}$ in other cancers, and does the efficacy of relevant endocrine therapies depend upon RB status? Early loss of RB is generally linked to responsiveness to conventional genotoxic therapies in various tumor types, but is the opposite true for endocrine therapy? For example, does RB loss mitigate against hormone therapy in breast cancer, as has been suggested previously (14)? Previous work has also identified a direct interaction between RB and the glucocorticoid receptor (15). Does this come into play in modulating stress responses in cancer etiology? Overall, the work of Sharma and colleagues (4) raises many intriguing questions that have major implications for how we diagnose and treat prostate cancer, but to what extent we must reconsider a role for
$\mathrm{RB}$ in the late stages of and for therapy of other human cancers remains to be seen.

\section{Acknowledgments}

The author's work is supported by NIH grant NCI-RO1-CA131188.

Address correspondence to: Kay F. Macleod, Ben May Department for Cancer Research, Gordon Center for Integrative Sciences, W-338, University of Chicago, 929 E. 57th Street, Chicago, Illinois 60637, USA. Phone: 773.834.8309; Fax: 773.702.4476; E-mail: kmacleod@uchicago.edu.

1. Burkhart DL, Sage J. Cellular mechanisms of tumour suppression by the retinoblastoma gene. Nat Rev Cancer. 2008;8(9):671-682.

2. Chen HZ, Tsai SY, Leone G. Emerging roles of E2Fs in cancer: an exit from cell cycle control. Nat Rev Cancer. 2009;9(11):785-797.

3. Okamoto A, et al. Mutations and altered expression of p16INK4a in human cancer. Proc Natl Acad Sci US A. 1994;91(23):11045-11049.

4. Sharma A, et al. The retinoblastoma tumor suppressor controls androgen signaling and human prostate cancer progression. J Clin Invest. 2010; 120(12):4478-4492.

5 . Huggins $\mathrm{CB}$, Hodges CV. Studies on prostatic cancer I: The effect of castration, of estrogen and of andro- gen injection on serum phsophatase in metastatic carcinoma of prostate. Cancer Res. 1941;1:293-297.

6. Shen MM, Abate-Shen C. Molecular genetics of prostate cancer: new prospects for old challenges. Genes Dev. 2010;24(18):1967-2000.

7. Taylor BS, et al. Integrative genomic profiling of human prostate cancer. Cancer Cell. 2010;18(1):11-22.

8. Wang QJ, et al. Androgen recpetor regulates a distinct transcription program in androgen-independent prostate cancer. Cell. 2009;138(2):245-256.

9. Foster CS, et al. Transcription factor E2F3 overexpressed in prostate cancer independently predicts clinical outcome. Oncogene. 2004;23(35):5871-5879.

10. Lee EY, Cam H, Ziebold U, Rayman JB, Lees JA, Dynlacht BD. E2F4 loss suppresses tumorigenesis in Rb mutant mice. Cancer Cell. 2002;2(6):463-472.

11. Ianari A, et al. Proapoptotic function of the retinoblastoma tumor suppressor protein. Cancer Cell. 2009;15(3):184-194.

12. Tran C, et al. Development of a second-generation antiandrogen for treatment of advanced prostate cancer. Science. 2009;324(5928):787-790.

13. Knudson AG. Mutation and cancer: statistical study of retinoblastoma. Proc Natl Acad Sci U S A. 1971;68(4):820-823.

14. Bosco EE, et al. The retinoblastoma tumor suppressor modifies the therapeutic response of breast cancer. J Clin Invest. 2007;117(1):218-228.

15. Singh P, Coe J, Hong W. A role for retinoblastoma protein in potentiating transcriptional activation by the glucocorticoid receptor. Nature. 1995; 374(6522):562-565

16. Weinberg RA. The retinoblastoma protein and cell cycle control. Cell. 1995;81(3):323-330.

\title{
Which species are in your feces?
}

\section{Colby Zaph}

Biomedical Research Centre, Department of Pathology and Laboratory Medicine, University of British Columbia, Vancouver, British Columbia, Canada.

\begin{abstract}
Nosocomial infections (i.e., infections acquired as a result of treatment in a hospital or health care unit) result in approximately 100,000 deaths and cost more than 25 billion dollars per year in the US alone. These infections are caused primarily by bacteria and affect mainly immunosuppressed patients. However, not all patients acquire infections, and the events leading up to infection are unclear. In this issue of the JCI, Ubeda et al. report how acquisition of one such infection, vancomycin-resistant Enterococcus faecium (VRE), is linked to a shift in the microbial flora following antibiotic treatment. This study highlights the potential for high-throughput sequencing of intestinal microbiota as a means to identify high-risk populations.
\end{abstract}

Nosocomial infections are infections acquired as a result of treatment in a hospital or health care unit. They are increasingly becoming a significant public health problem. It is thought that $5 \%-10 \%$ of patients entering hospitals worldwide develop a hospital-acquired infection (HAI) (1). The global growth of antibiotic-resistant bacterial strains, such as methicillin-resistant

Conflict of interest: The author has declared that no conflict of interest exists.

Citation for this article: J Clin Invest. 2010; 120(12):4182-4185. doi:10.1172/JCI45263.
Staphylococcus aureus (MRSA) and vancomycin-resistant Enterococcus faecium (VRE), has complicated the control of HAIs and highlights the need for a better understanding of how HAIs develop, are acquired, and may be controlled.

Intestinal Enterococcus infections are among the most common type of infection acquired by hospitalized patients. While enterococcal infections are much less capable of causing disease than HAIs such as Clostridium difficile and S. aureus, they still cause complications, prolong hospital stays, and can be fatal, particularly those caused by VRE. Fatal VRE infections, which occur upon bacterial invasion of the bloodstream, primarily affect immunocompromised patients such as those undergoing bone marrow or hematopoietic stem cell transplantation (HSCT) (2). It has been estimated that up to $50 \%$ of HSCT patients acquire VRE, and infection with VRE is independently associated with increased mortality in these patients (3). Identifying high-risk patients and developing novel methods to combat VRE are critical components in combating HAIs. The study by Ubeda et al. in this issue of the JCI begins to address this in both mice and humans using high-throughput sequencing analysis of commensal flora populations (4).

\section{Commensal flora and susceptibility to infection}

Over the past several years, interest in understanding the relationship between the commensal flora and disease states has increased markedly. It is clear that changes in the flora have a dramatic effect on the 